\title{
Failed detection of complex congenital heart disease (including double outlet right ventricle and total anomalous pulmonary venous return) by neonatal pulse oximetry screening
}

\author{
Patrick G. Hetzel • René Glanzmann • Joëlle Günthard • \\ Elisabeth Bruder • Esther Godi • Christoph Bührer
}

Received: 6 April 2006 /Revised: 28 July 2006 / Accepted: 2 August 2006 / Published online: 19 September 2006

(C) Springer-Verlag 2006

\begin{abstract}
We report on a newborn infant with complex congenital heart disease (CHD) featuring double outlet right ventricle and hypoplastic left ventricle who had postductal oxygen saturation well above $95 \%$ and thus eluded pulse oximetry screening for CHD.
\end{abstract}

Keywords Complex heart defect .

Double outlet right ventricle $\cdot$ Hypoplastic left ventricle

Neonatal pulse oximetry screening

After an uneventful pregnancy with unsuspicious first and second trimester out-of-hospital ultrasound scans, this girl was born at term from meconium-stained amniotic fluid with Apgar scores of 9, 10 and 10. She was transferred to the neonatology ward at $15 \mathrm{~h}$ of age because of tachypnea. There

P. G. Hetzel · R. Glanzmann • C. Bührer

Department of Neonatology, University Children's Hospital,

Basel, Switzerland

J. Günthard

Department of Cardiology, University Children's Hospital,

Basel, Switzerland

E. Bruder

Department of Pathology, University Hospital,

Basel, Switzerland

E. Godi

Department of Obstetrics, Women's Hospital Bruderholz, Basel, Switzerland

P. G. Hetzel $(\bowtie)$

Universitäts-Kinderspital beider Basel,

CH-4005 Basel, Switzerland

e-mail: patrick.hetzel@ukbb.ch was no heart murmur, pulses were equally palpable, and upper and lower extremities blood pressure was normal. Chest Xrays showed some minor pulmonary opacities, heart was normal by size and shape. She was transiently given supplemental oxygen (maximum 40\%) during the first $9 \mathrm{~h}$. Thereafter, pre-and postductal oxygen saturations (HewlettPackard HP66S) were consistently above 95\% also with room air. Blood gas analysis was always normal (minimum $\mathrm{pH}$ 7.34, worst base deficit-5 mmol/l). Tachypnea persisted until day 9 of life when the infant suddenly had progressive cyanosis (minimum oxygen saturation 84\%) responsive to supplemental oxygen, apneas, absence of femoral pulses, hypotension of the lower extremities, oliguria and metabolic acidosis. Echocardiography revealed complex CHD, including a double outlet right ventricle, total anomalous pulmonary venous return into the superior vena cava, primum-type atrial septal defect, mitral valve atresia, hypoplastic left ventricle, coarctation, and patent ductus arteriosus. After discussions with the cardiologists and neonatologists involved, the parents decided against surgery, and the girl died soon afterwards. Autopsy confirmed the echocardiographic findings.

A recent report in this journal demonstrated that postductal pulse oximetry screening had $100 \%$ sensitivity and $99.7 \%$ specifity for detection of critical CHD [2]. Out of 3,262 asymptomatic newborn infants, pulse oximetry screening identified 17 infants with critical CHD, while there were no false negative cases. Based on this study, the Swiss Societies of Pediatric Cardiology and Neonatology have recommended general screening by pulse oximetry [1].

Some critical CHD (such as coarctation with large ventricular septal defect or critical aortic stenosis) may go undetected if there is abundant central right-to-left shunt [3-6]. 
Moreover, CHD with common outlet, such as double outlet ventricle (left or right) may show equal and even normal oxygen saturation in both great arteries if pulmonary blood flow greatly exceeds systemic blood flow. Thus, pulmonary overcirculation may mask CHD by normal saturations and tachypnea may remain the only clinical sign.

In our case, two hemodynamic peculiarities contributed to regular postductal saturations: 1. Pre-atrial mixing of oxygenated and desoxygenated blood (total anomalous pulmonary venous return) with high saturation in the right atrium and ventricle. 2. Origin of both great arteries from the right ventricle. Clinical deterioration ensued following narrowing of the duct and the aortic isthmus with diminished perfusion of the lower body half.

Our case emphasises that pulse oximetry screening, even when used in conjunction with clinical examination, may miss critical CHD. Persistent unexplained tachypnea in newborn infants should prompt echocardiographic examination.

\section{References}

1. Arlettaz R, Bauersfeld U (2005) Empfehlungen zum neonatalen Screening angeborener Herzfehler. Paediatrica 16:34-37

2. Arlettaz R, Bauschatz AS, Mönkhoff M, Essers B, Bauersfeld U (2006) The contribution of pulse oximetry to the early detection of congenital heart disease in newborns. Eur J Pediatr 165:9498

3. Koppel RI, Druschel CM, Carter T, Goldberg BE, Mehta PN, Talwar R, Bierman FZ (2003) Effectiveness of pulse oximetry screening for congenital heart disease in asymptomatic newborns. Pediatrics 111:451-455

4. Reich JD, Miller S, Brogdon B, Casatelli J, Gompf TC, Huhta JC, Sullivan K (2003) The use of pulse oximetry to detect congenital heart disease. J Pediatr 142:268-272

5. Richmond S, Reay G, Abu-Harb M (2002) Routine pulse oximetry in the asymptomatic newborn. Arch Dis Child Fetal Neonatal Ed 87:F83-F88

6. Rosati E, Chitano G, Dipaola L, De Felice C, Latini G (2005) Indications and limitations for a neonatal pulse oximetry screening of critical congenital heart disease. J Perinat Med $33: 455-457$ 